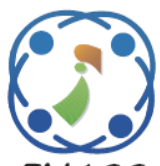

\title{
A PAPR Reduction in FBMC-OQAM System via Overlapping-PTS with Artificial Bee Colony Phase Optimization
}

\author{
Panya Jirajaracheep \\ Tanairat $\operatorname{Mata}^{2 *}$ \\ Pisit Boonsrimuang ${ }^{1}$ \\ ${ }^{I}$ Faculty of Engineering, King Mongkut's Institute of Technology Ladkrabang, Bangkok, 10520 Thailand \\ ${ }^{2}$ Faculty of Engineering, Rajamangala University of Technology Krungthep, Bangkok, 10120 Thailand \\ * Corresponding author's Email: tanairat.m@mail.rmutk.ac.th
}

\begin{abstract}
In this paper, the overlapping Partial Transmit Sequence (overlapping-PTS) with Artificial Bee Colony $(\mathrm{ABC})$ phase optimization method is proposed to reduce the larger Peak-to-Average Power Ratio (PAPR) which is the major drawback of filter bank multicarrier with offset quadrature amplitude modulation (FBMC-OQAM) system. In this proposed method, we clarify the problem of larger PAPR by reducing the PAPR of each overlapping data symbol with lower combination of the optimal phase factors. Unlike the conventional PTS methods, the proposed method uses the lower combination of phase factors by applying the $\mathrm{ABC}$ algorithm with considering the overlap between the current data symbol and the past data symbol to search the optimal phase combination. The proposed overlapping-PTS with $A B C$ phase optimization method can significantly reduce the larger PAPR with low computational complexity for the FBMC-OQAM system which can be verified by the computer simulation results. From the computer simulation results, it can be confirmed that the proposed method shows much better PAPR which can reduce the PAPR by approximately $1.9 \mathrm{~dB}$ at CCDF $10^{-1}$ as comparing with the conventional PTS method and can perform much lower computational complexity than the conventional methods which are only $4.38 \%$ and $0.76 \%$ for $S$ phase factor pattern $=300$ and 50 respectively.
\end{abstract}

Keywords: High PAPR, FBMC-OQAM, Partial transmit sequence, Overlapping, ABC phase optimization.

\section{Introduction}

By using the orthogonal frequency division multiplexing (OFDM) technique $[1,2]$ which is one of the multicarrier modulation techniques in the wireless communication system, efficient usage of the spectral frequency and an eliminated ISI through the use of GI insertion in the multipath channel can be performed. However, the efficiency of spectra and power would be decreased by adding the guard interval (GI). To realize this issue, the filter bank multicarrier with offset quadrature amplitude modulation (FBMC-OQAM) technique is applied for solving which can increase the efficiency of the spectra with an expense of computational complexity and decrease the leakage of out-of-band power [3].

In the $5 \mathrm{G}$ mobile communication standard, the FBMC-OQAM is considered for a candidate because of its advantages such as the good time and frequency localization, low out-of-band radiation, robustness to phase noise and so on [4, 5]. A prototype filter is used in the FBMC-OQAM technique [6] for reducing the orthogonality of subcarriers instead of GI insertion in OFDM technique which can achieve higher spectral efficiency. From the explosive increase in demand for mobile and wireless broadband services in $5 \mathrm{G}$, the FBMC-OQAM technique could be helper effectively for the OFDM technique [7].

However, the major drawback of both OFDM and FBMC-OQAM as the multicarrier modulation techniques is the larger Peak-to-Average Power Ration (PAPR). From this problem, the higher nonlinear signal distortion has occurred at the output of a power amplifier which leads to the fatal degradation of bit-error-rate (BER) performance. To solve this problem, many PAPR reduction methods 


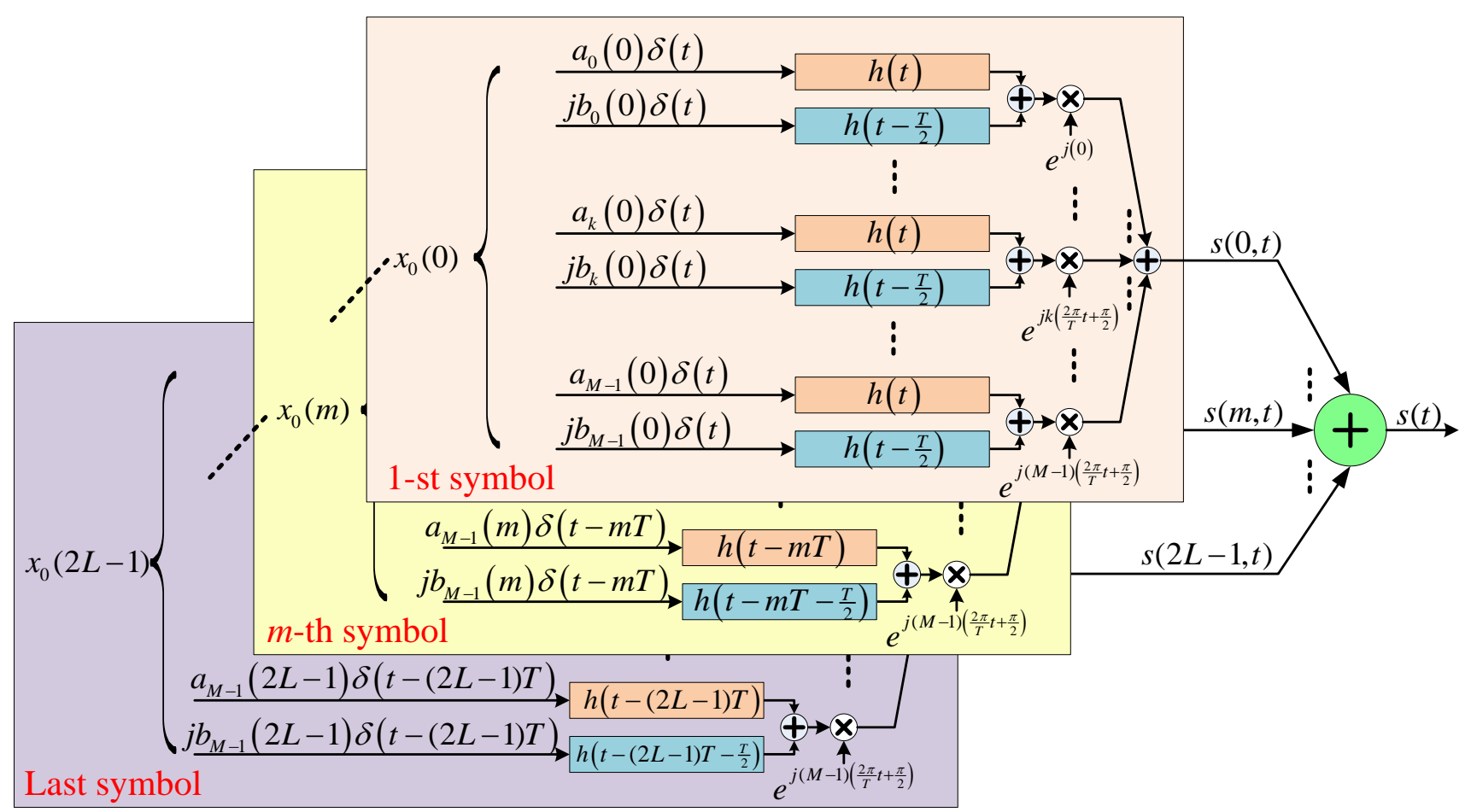

Figure. 1 A structure of transmitter in the FBMC-OQAM system

have been proposed for the multicarrier modulation systems such as Partial Transmit Sequence (PTS) [8, 9], Selective Mapping (SLM) [10] and so on.

The PTS method which is one of the popular PAPR reduction methods is known as a distortionless technique. In the PTS method, the minimum PAPR value will obtain from the multiplication between data signal cluster of each data symbol and the optimal phase combination. The side information sent from the transmitter is required at the receiver for data demodulation correctly. In this method, the computational complexity of optimizing phase combination process is increasing proportionally to the number of clusters expanding. In [11], the PTS method was applied for the FBMC-OQAM system. From the results in [11], the PAPR performance of FBMCOQAM system can be improved by using the PTS method insignificantly. From this result, it is difficult to get sufficient PAPR performance by applying the PTS method in the FBMC-OQAM system directly due to the overlapping symbol structure of FBMC-OQAM system. Therefore, the PAPR of each data symbol cannot be optimized independently like that in the OFDM system.

Furthermore, the PTS method has also produced the huge computational complexity in the search of the optimal phase combination. Many solutions to this problem have been proposed such as flipping iteration technique [12], phase coefficient searching technique [13], PTS with artificial bee colony $(\mathrm{ABC})$ technique $[14,15]$ and so on. One of the promising techniques among these techniques is PTS-ABC technique which can reduce the PAPR performance with low computational complexity especially when increasing the number of clusters.

In this paper, we propose a solution to the larger PAPR problem in the FBMC-OQAM system which can perform better the PAPR performance and produce lower the computational complexity of optimizing phase combination than that of using the conventional PTS method. The silent features are to minimize the larger PAPR by applying the overlapping-PTS method [16] and to optimize the phase combination by using the artificial bee colony (ABC) phase optimization. Similar to the PTS method, the receiver requires the transmitter to send the side information for data demodulation.

This paper is organized as follows: Section 2 introduces the definition of PAPR for the FBMCOQAM signals. Section 3 proposes the overlappingPTS technique with $\mathrm{ABC}$ phase optimization for FBMC-OQAM system. The performance evaluations of the proposed and the other methods for PAPR reduction are presented in Section 4. Finally, Section 5 draws some conclusions. 


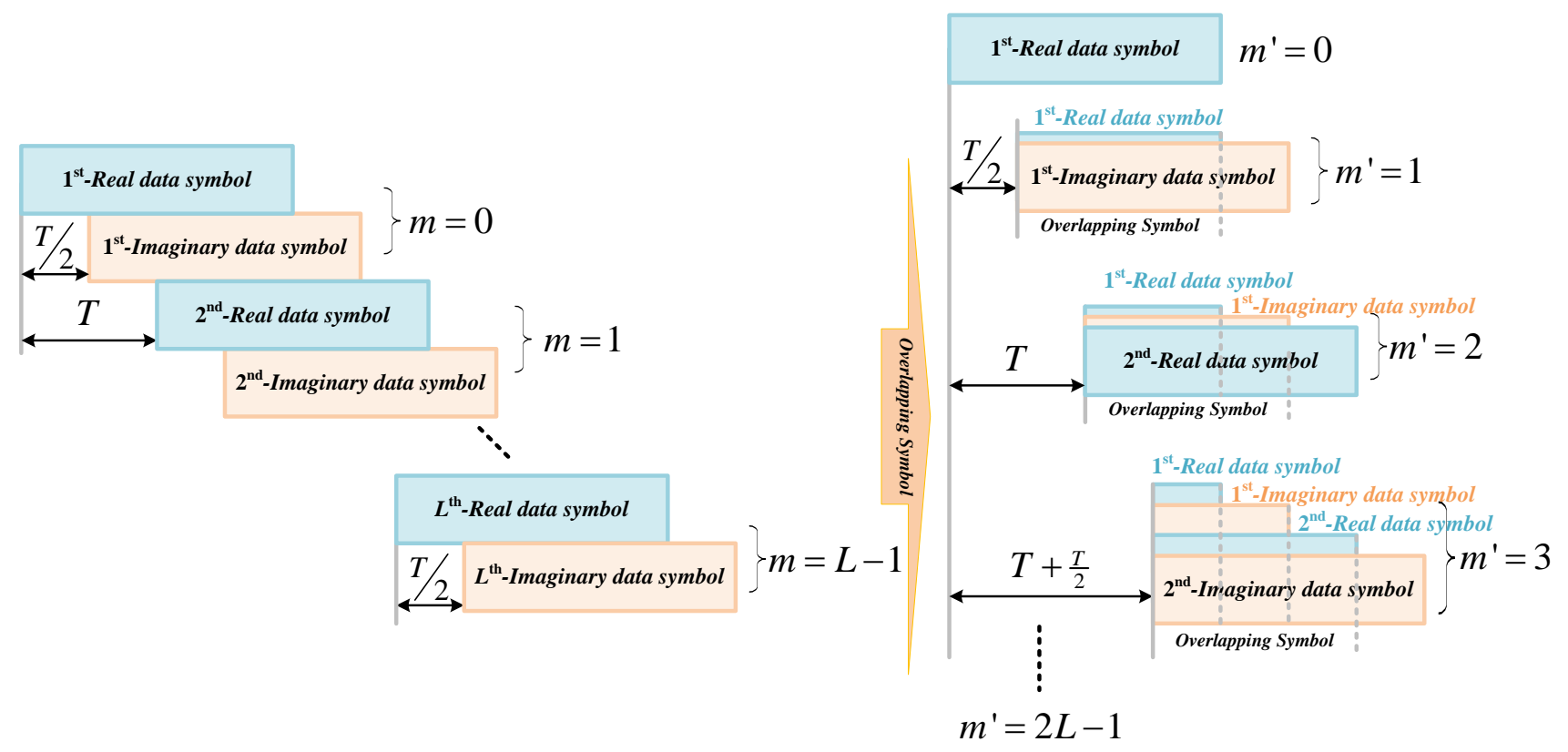

Figure. 2 The overlapping data symbol in FBMC-OQAM system

\section{FBMC-OQAM system and PAPR}

Fig. 1 shows the overview of transmitter structure in the FBMC-OQAM system. The input data $X_{m}(m),(n=0,1,2, \ldots, N$-1 $)$ at $n$-th subcarrier and $m$-th symbol will be modulated by OQAM modulation and transformed by IFFT with $N$-point which the modulation complex data symbols can be expressed by,

$$
x_{k}(m)=a_{k}(m)+j b_{k}(m), 0 \leq k \leq N-1,
$$

where $a$ is the real part as in-phase (I) component and $b$ is imaginary part as quadrature (Q) component which I and $\mathrm{Q}$ components are stepped by $T / 2$ in time-domain where $K$ is the overlapping factor which is the ratio of impulse response $h(t)$ duration to the multicarrier symbol period $(T)$. Then, $x_{k}(m)$ will be passed through a prototype filter with $h(t)$ and $T / 2$ carrier frequency spacing which can be given by,

$$
\begin{aligned}
s(t)=\sum_{k=0}^{M-1} \sum_{m=0}^{2 L-1}\left\{a_{k}(m) h(t-m T)\right. & \\
& +j b_{k}(m) h\left(t-m T-\frac{T}{2}\right) e^{j k\left(\frac{2 \pi t}{T}+\frac{\pi}{2}\right)} .
\end{aligned}
$$

A PHYDYAS [6] as a prototype filter is designed for reducing the out-of-band leakage in the FBMC-OQAM system. It should be noted that the filter impulse response is spanned by 4 times of the multicarrier symbol period.

In this paper, the Solid State Power Amplifier (SSPA) [17] with AM/AM conversion is assumed as the non-linear amplifier which can be expressed the relationship between input and output by the following equation,

$$
F[\rho]=\frac{\rho}{\left[1+\left(\frac{\rho}{A}\right)^{2 r}\right]^{1 / 2 r}},
$$

where $\rho$ and $A$ denote the amplitude of the input signal and the saturated output level respectively. The decision of non-linear amplifier level is represented by $r$. The operation point in the nonlinear amplifier is defined by the input back-off (IBO) which is expressed as,

$$
\left.I B O\right|_{d B}=10 \log \left(\frac{P_{\text {input }}}{P_{\text {output }}}\right) .
$$

Generally, the PAPR for the multicarrier signal is defined as the ratio of the maximum transmitted signal power to the average transmitted signal power which can be given by the following equation,

$$
\left.P A P R\right|_{d B}=10 \log _{10}\left(\frac{\max _{0 \leq t \leq T}|s(t)|^{2}}{\frac{1}{T} \int_{0}^{T}|s(t)|^{2} d t}\right) .
$$

In the evaluation of PAPR performance, the PAPR reduction will be observed from the probability of PAPR over a PAPR reference $\left(\mathrm{PAPR}_{0}\right)$ as the Complementary Cumulative Distribution function (CCDF) which can be written as,

$$
C C D F(P A P R)=\operatorname{Pr}\left(P A P R>P A P R_{0}\right) .
$$




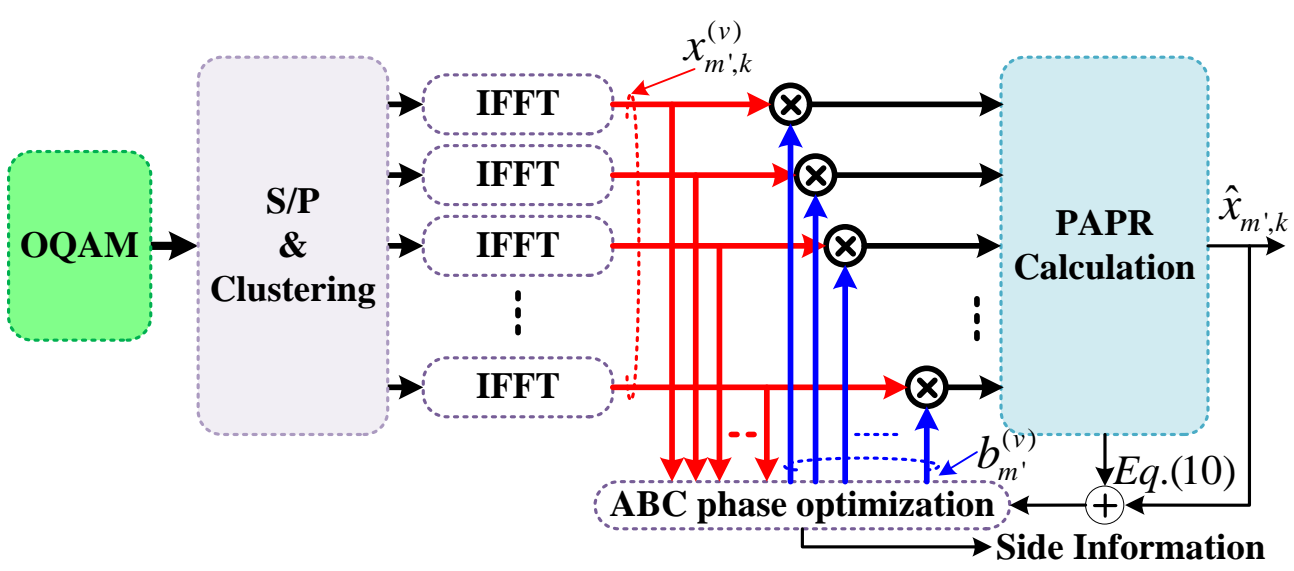

Figure. 3 The block diagram of the proposed PAPR reduction method

\section{Proposal of overlapping-PTS with ABC phase optimization method for FBMC- OQAM system}

From the structure of FBMC-OQAM symbol as shown in Fig.2, the symbols will be overlapped with $K M$ symbol length. The next symbol $L$-th always overlaps with the previous symbol $(L-1)$-th. Every imaginary data symbol is delayed $T / 2$ of its real data symbol. From this reason, it is not enough PAPR reduction considering in each data symbol independently for FBMC-OQAM symbol. To satisfy the requirement, this paper proposes the PAPR reduction for FBMC-OQAM system by modifying the conventional PTS as the overlapping-PTS method in this paper.

\subsection{Overlapping-PTS technique}

From the Fig.2, the structure of overlapping data symbol is utilized in the proposed method. Like a conventional PTS method, the first data symbol $(m$ ' $=0$ ) without overlapping any adjacent data symbol is divided into $V$ cluster and converted from frequency domain into time domain by using IFFT with $N$ points as $x_{0, k}^{(v)}$. The first data symbol signal $\hat{x}_{0, k}^{(v)}$ with the optimal PAPR can be expressed as,

$$
\hat{x}_{0, k}=\sum_{v=1}^{V}\left(b_{0}^{(v)} x_{0, k}^{(v)}\right),
$$

where $b^{(v)}\left(=e^{j \phi^{(v)}}\right)$ is the phase weighting factor at $v$-th cluster and $\phi^{(v)} \in \frac{2 \pi i}{W} \mid i=0, \cdots, W-1 . W$ is the number of predetermined phases. The optimal PAPR of the first data symbol can be defined by,

$$
\left[b_{0}^{(1)}, b_{0}^{(2)}, \ldots, b_{0}^{(V)}\right]=\min _{w}\left(\max _{k}\left(\left|\sum_{v=1}^{V}\left(b_{0}^{(v)} x_{0, k}^{(v)}\right)\right|^{2}\right)\right) \text {. }
$$

Next, the overlapping data symbols started from the second $\left(m^{\prime}=0\right)$ to the last $\left(m^{\prime}=2 L-1\right)$ data symbols which can be given by,

$$
\left.\hat{x}_{m^{\prime}, k}\right|_{m^{\prime} \neq 0}=x_{\left(m^{\prime}-1\right),\left(k+\frac{T}{2}\right)}+\sum_{v=1}^{V}\left(b_{m^{\prime}}^{(v)} x_{m^{\prime}, k}^{(v)}\right) .
$$

From (9), it can be optimized the PAPR which is chosen from,

$$
\begin{array}{r}
{\left[b_{m^{\prime}}^{(1)}, b_{m^{\prime}}^{(2)}, \ldots, b_{m^{\prime}}^{(V)}\right]} \\
=\min _{w}\left(\max _{k}\left(\left|x_{\left(m^{\prime}-1\right),\left(k+\frac{T}{2}\right)}+\sum_{v=1}^{V}\left(b_{m^{\prime}}^{(v)} x_{m^{\prime}, k}^{(v)}\right)\right|^{2}\right)\right), \\
1 \leq m^{\prime} \leq 2 L-1 .
\end{array}
$$

Fig. 3 shows the block diagram of the proposed overlapping-PTS with $\mathrm{ABC}$ phase optimization method.

\subsection{Minimization of PAPR by using ABC phase optimization}

To obtain the FBMC-OQAM signal with the optimal PAPR, the huge computational complexity by using the conventional PTS method is solved by the proposed artificial bee colony (ABC) phase optimization [14-15]. The proposed ABC phase optimization method can achieve better PAPR performance with the lower computational complexity.

In the main concept of $\mathrm{ABC}$ algorithm, the position of a food source is the possible solution of optimization problem which is solved by employed bees, onlooker bees and scout bees in the colony of artificial bees. In the PAPR reduction problem by using the $\mathrm{ABC}$ phase optimization, the position of a food source is represented by a phase weighting 


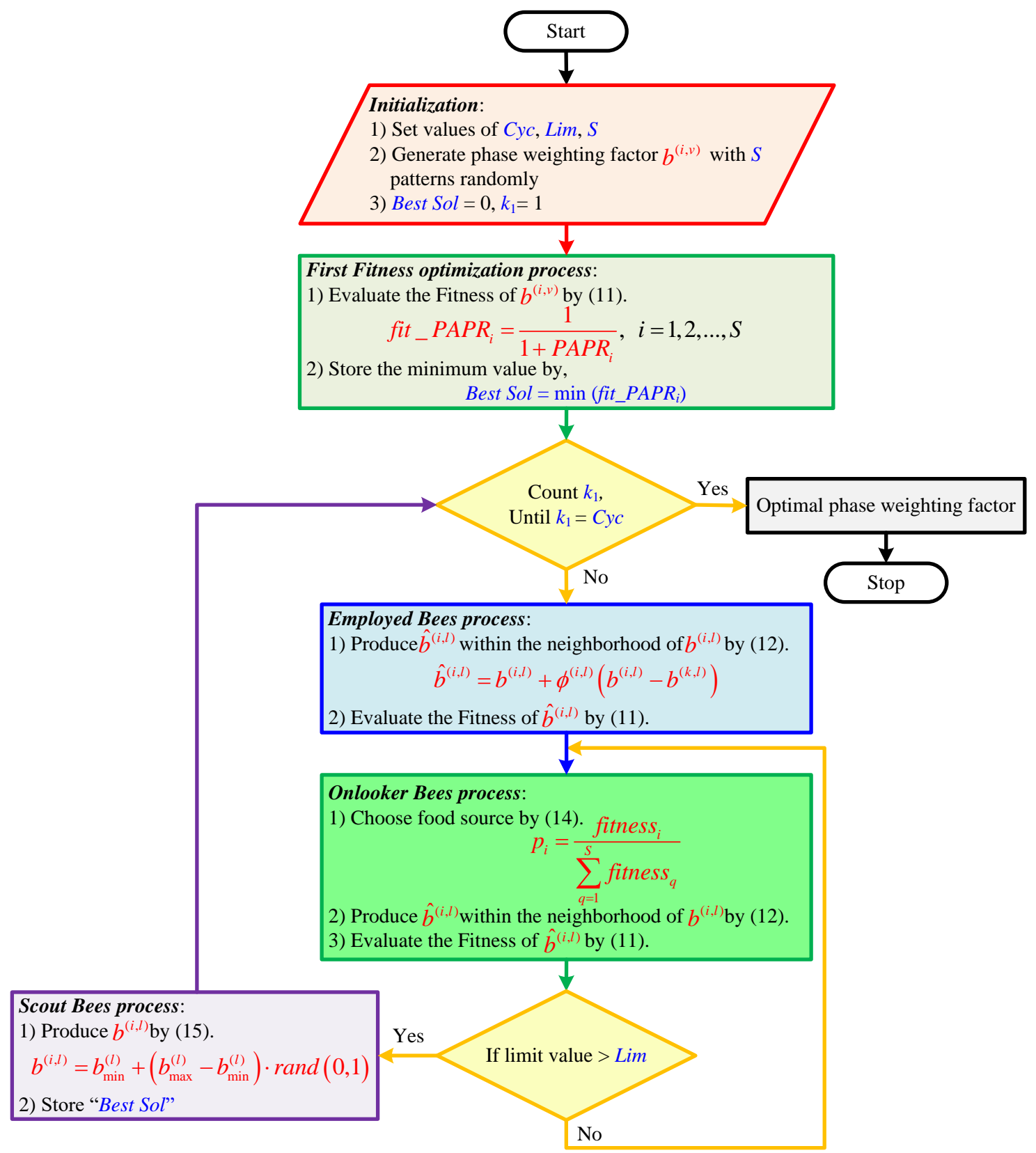

Figure. 4 The flowchart of the ABC phase optimization process

factor $b^{(i, v)}$ with the number of phase weighting factor patterns $(S)$ where $i=1,2, \ldots, S$. In the ABC phase optimization, there are four sections the following as,

Section 1: this section is the initialization, the random phase weighting factor $b^{(i, v)}$ is generated initially then the fitness value of $b^{(i, v)}$ within the phase weighting factor patterns will be evaluated by the following equation,

$$
\operatorname{fitness}\left(b^{(i, v)}\right)=\frac{1}{1+\operatorname{PAPR}\left(\sum_{v}\left(b^{(i, v)} \cdot x_{k}^{(v)}\right)\right)} .
$$

Section 2: the new phase weighting factor $\hat{b}^{(i, l)}$ as a new food source is generated within the neighbourhood of $b^{(i, l)}$ by the employed bees which can be produced by,

$$
\begin{aligned}
\hat{b}^{(i, l)} & =b^{(i, l)}+\phi^{(i, l)}\left(b^{(i, l)}-b^{(k, l)}\right), \\
l & \in\{1,2, \ldots, S\} \& k \in\{1,2, \ldots, J\} \text { where } i \neq k,
\end{aligned}
$$

where $J$ is the number of employed bees and $\phi$ is a random number in the range $[-1,1]$. Because the $\hat{b}^{(i, l)}$ is the discrete phase, $\hat{b}^{(i, l)}$ as given by (11) for $W=2$ can be selected by the following condition, 


$$
\hat{b}^{(i, l)}=\left\{\begin{array}{lc}
1, & \text { if } \frac{\pi}{4} \leq \hat{b}^{(i, l)}<\frac{5 \pi}{4} \\
-1, & \text { else }
\end{array}\right.
$$

Section 3: the onlooker bees will select a food source depending on the probability value associated with that food source $p_{i}$ which can be calculated by the following equation,

$$
p_{i}=\frac{\text { fitness }_{i}}{\sum_{q=1}^{S} \text { fitness }_{q}} .
$$

Section 4: after completed the process in Section 2 and 3, if there is no any improvement of the fitness values of the food source with the limit value (Lim), the employed bees become the scout bees. The scout bees produce the new food sources randomly by,

$$
b^{(i, l)}=b_{\min }^{(l)}+\left(b_{\max }^{(l)}-b_{\min }^{(l)}\right) \cdot \operatorname{rand}(0,1) .
$$

From Sections 1-4, the following explain the PAPR reduction procedures of the proposed overlapping-PTS with $\mathrm{ABC}$ phase optimization by using Fig.4.

\section{Results and Discussions}

In this section, this paper shows the PAPR reduction and BER performance of the proposed overlapping-PTS with $\mathrm{ABC}$ phase optimization which are evaluated by the computer simulation using the parameters listed in Table 1 .

Fig. 5 shows the PAPR value of the proposed method with different $S$ phase factor patterns when changing a number of Cycles $(C y c)$. Here, $\operatorname{Lim}=5$ and different $S=20,30$ and 40, respectively. From the result, it can be seen the PAPR of all is decreasing rapidly for $C y c=1$ to $C y c=5$ after that its change becomes slight. Furthermore, it can be also observed that the PAPR for $S=40$ is better than that of both $S=20$ and 30, respectively. From the result, the following evaluations for the PAPR performance employs $\operatorname{Lim}=5$ and $C y c=5$ for the proposed method in this paper. Meanwhile, Fig.6 shows the PAPR value of the proposed method with $\operatorname{Lim}=5$ and $C y c=5$ when changing size of $S$ phase factor patterns. From the result, it can be confirmed that the PAPR value can be reduced by increasing $S$ phase factor patterns. However, its computational complexity would be increased which corresponds to the increasing of $S$ phase factor patterns.
Table 1. Simulation parameters

\begin{tabular}{|c|c|}
\hline Parameters & Values \\
\hline Technique & FBMC-OQAM \\
\hline Modulation with Gray coding & OQPSK \\
\hline Demodulation & Coherent \\
\hline OFDM occupied bandwidth & $5 \mathrm{MHz}$ \\
\hline$N$-data subcarriers & 64 \\
\hline$V$-cluster & 16 \\
\hline$W$-discrete phase & 2 \\
\hline$K$-overlapping factor & 4 \\
\hline Prototype filter type & PHYDYAS [6] \\
\hline \multirow{2}{*}{ Non-linear amplifier with IBO(dB) } & SSPA with - \\
\hline$r$-SSPA non-linear parameter & 2 \\
\hline
\end{tabular}

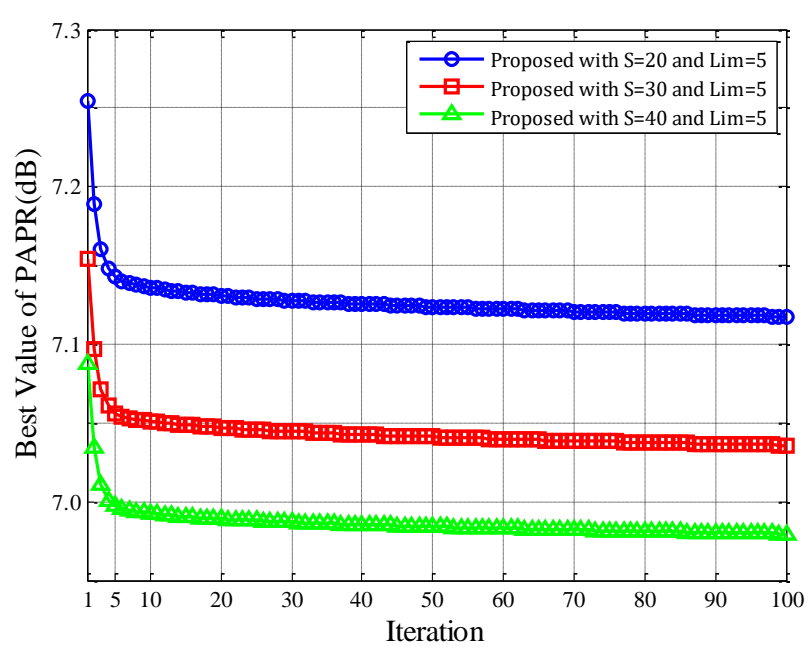

Figure. 5 PAPR values with different $S$ phase factor patterns when changing Cycle number

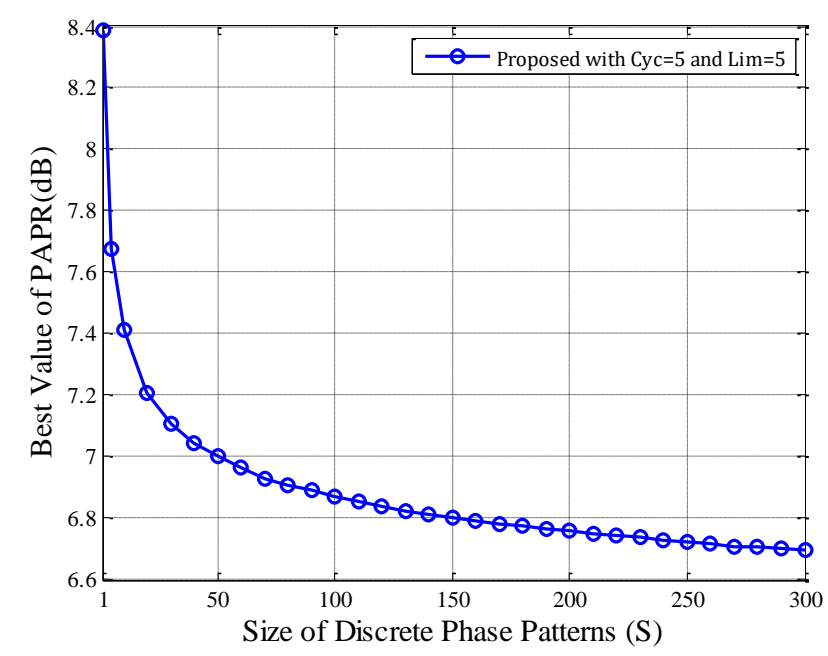

Figure. 6 PAPR value vs $S$ phase factor pattern when $\mathrm{Cyc}=5$ and $\operatorname{Lim}=5$ 


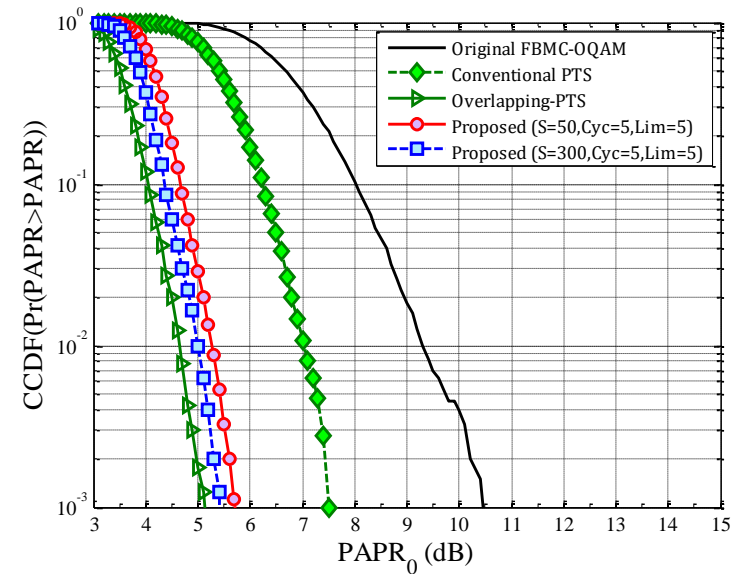

Figure. 7 PAPR performance for the proposed method

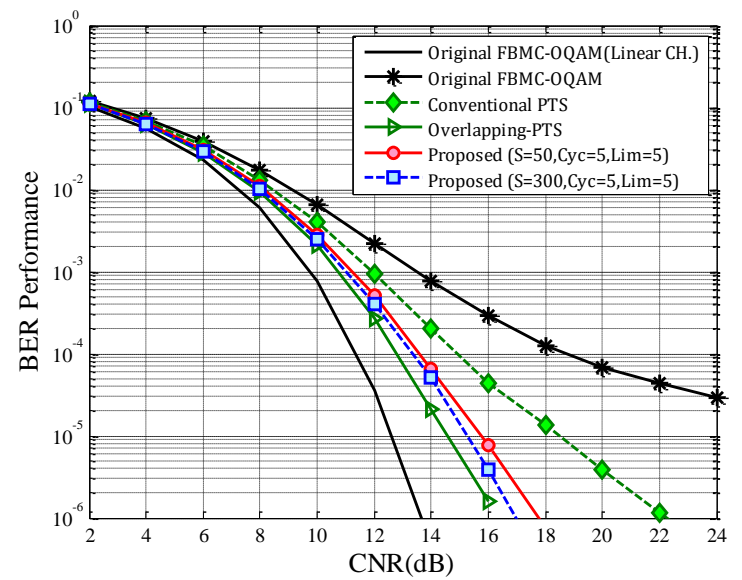

Figure. 8 BER performance for the proposed method in the non-linear channel (IBO=-2dB.)

Table 2. Comparison of computational complexity for the proposed, Conventional PTS and Overlapping-PTS methods at $\mathrm{CCDF}=10^{-1}[14]$

\begin{tabular}{|c|c|c|c|}
\hline $\begin{array}{c}\text { PAPR Reduction Method (V=16 } \\
\text { and } \mathrm{W}=2)\end{array}$ & PAPR & Computational Complexity & $\begin{array}{c}\text { Improvement from } \\
\text { Conventional PTS and } \\
\text { Overlapping-PTS }\end{array}$ \\
\hline Conventional PTS & $6.25 \mathrm{~dB}$ & $W^{(V-1)}=2^{(16-1)}=32,768$ & $100 \%$ \\
\hline Overlapping-PTS & $4.05 \mathrm{~dB}$ & $W^{(V-1)}=2^{(16-1)}=32,768$ & $100 \%$ \\
\hline Proposed with $S=300$ and $C y c=5$ & $4.35 \mathrm{~dB}$ & $S \times C y c=300 \times 5=1,500$ & $4.58 \%$ \\
\hline Proposed with $S=50$ and $C y c=5$ & $4.65 \mathrm{~dB}$ & $S \times C y c=50 \times 5=250$ & $0.76 \%$ \\
\hline
\end{tabular}

Fig. 7 shows the PAPR performance based on the CCDF for the FBMC-OQAM with the proposed overlapping-PTS with ABC phase optimization $(C y c$ $=5$ and $\operatorname{Lim}=5$ ). The performances of FBMCOQAM with two conventional PAPR reduction methods such as the PTS method [11] and the overlapping-PTS method [16] are also shown as the purpose of comparison with the proposed method. At $\mathrm{CCDF}=10^{-1}$, the PAPR of using overlapping-PTS method [16] is $4.05 \mathrm{~dB}$ approximately. Meanwhile, the PAPR of using the proposed method with $S=$ 300 and $S=50$ are approximately $4.35 \mathrm{~dB}$ and 4.65 $\mathrm{dB}$, respectively. When comparing with the PAPR of using overlapping-PTS method [16], although there are the different levels $0.3 \mathrm{~dB}$ and $0.6 \mathrm{~dB}$ respectively, the computational complexity of phase optimization process of using the proposed method is only $4.48 \%$ for $S=300$ and $0.76 \%$ for $S=50$ as shown in Table 2, respectively. Moreover, from the result in Fig.7, it can be seen that the proposed method shows the better PAPR performances withlow computational complexity than two conventional PAPR reduction methods $[11,16]$.

Fig. 8 shows the BER performance evaluated for the proposed method with different $S$ phase factor patterns when changing the carrier power to noise power ratio $(\mathrm{CNR})$ in the non-linear channel. In the simulation, the IBO of SSPA is taken by $-2 \mathrm{~dB}$. From the result, it can be seen that the proposed methods with $S=50$ and $S=300$ show much better BER performance than the original FBMC-OQAM and conventional PTS method [11] in the non-linear channel. Moreover, it can be observed that the BER performance of the proposed methods with low computational complexity is close to that of the overlapping-PTS method [16].

\section{Conclusions}

In this paper, we propose an overlapping-PTS with $A B C$ phase optimization method for the FBMC-OQAM system. The silent features of the proposed PAPR reduction method are to improve the PAPR by using the overlapping-PTS method and to reduce the computational complexity of phase optimization process by applying the ABC phase optimization. Compared to the existing PAPR reduction methods, the proposed method can achieve better PAPR performance which can reduce the PAPR by $1.9 \mathrm{~dB}$ approximately as comparing with the conventional PTS method [11] at CCDF 10 1 and can perform computational complexity lower than that of performing by using the conventional PAPR reduction methods $[11,16]$ which are only $4.38 \%$ and $0.76 \%$ for $S=300$ and 50 respectively. 
Furthermore, the proposed method can be adjusted the PAPR value easily by using the three control parameters such as $S$ phase factor patterns, Cycle number $(\mathrm{Cyc})$ and limit value $(\mathrm{Lim})$. From the computer simulation results, it can be confirmed that the proposed PAPR reduction method has the capability to provide the better PAPR performance with low computational complexity for FBMCOQAM system.

\section{References}

[1] T. Hwang, C. Yang, G. Wu, S. Li, and G.Y. Li, "OFDM and Its Wireless Applications: A Survey", IEEE Transactions on Vehicular Technology, Vol.58, No.4, pp.1673-1694, 2009.

[2] P. Aggarwal and V.A. Bohara, "A Nonlinear Downlink Multiuser MIMO-OFDM Systems", IEEE Wireless Communications Letters, Vol.6, No.3, pp.414-417, 2017.

[3] H.Q. Wei and A. Schmeink, "Comparison and Evaluation between FBMC and OFDM Systems", In: Proc. of International ITG Workshop on Smart Antennas, pp.1-7, 2015.

[4] P. Banelli, S. Buzzi, G. Colavolpe, A. Modenini, F. Rusek, and A. Ugolini, "Modulation Formats and Waveforms for 5G Networks: Who will be the heir of OFDM?: An Overview of Alternative Modulation Schemes for Improved Spectral Efficiency", IEEE Signal Processing Magazine, Vol.31, No.26, pp.80-93, 2014.

[5] F.L. Lua and Z. Charlie, "Signal Processing for 5G Algorithms and Implementations", Wiley IEEE Press, 2016.

[6] S. Frank, "Filterbank Based Multi Carrier Transmission (FBMC) - Evolving OFDM", In: Proc. of European Wireless Conference, pp.1051-1058, 2010.

[7] F. Schaich and T. Wild, "Waveform Contenders for 5G OFDM vs FBCM vs UFMC", In: Proc. of International Conf. Symposium on Communications, Control and Signal Processing, pp.457-460, 2014.

[8] Y.A. Jawhar, L. Audah, M.A. Taher, K.N. Ramli, N.S.M. Shah, M. Musa, and M.S. Ahmed, "A Review of Partial Transmit Sequence for PAPR Reduction in the OFDM Systems", IEEE Access, Vol.7, pp.18021-18041, 2019.

[9] J.H. Moon, Y.R. Nam, and J.H. Kim, "PAPR Reduction in the FBMC-OQAM System via Segment-Based Optimization", IEEE Access, Vol.6, pp.4994-5002, 2018.

[10] A. Mohammed, S. Hussein, R. Amr, and A. Saleh, "A Novel Iterative-SLM Algorithm for PAPR Reduction in 5G Mobile Fronthual
Architecture", IEEE Photonics Journal, Vol.11, No.1, pp.1-12, 2019.

[11] D. Honggui, R. Shuang, L. Yan, and T. Chengying, "Modified PTS-based PAPR Reduction for FBMC-OQAM Systems", Journal of Physics Conference Series, Vol.910, No.1, pp.1-8, 2017.

[12] J.C. Leonard and R.S. Nelson, "Peak-toAverage Power Ratio Reduction of an OFDM Signal Using Partial Transmit Sequences", IEEE Communications Letter, Vol.4, No.3, pp.86-88, 2000.

[13] P. Pantiko, T. Mata, P. Boonsrimuang, and H. Kobayashi, "A Low Complexity Improved-PTS Phase Coefficient Searching Algorithm for OFDM System", In: Proc. of International Conf. on Electrical Engineering/Electronics, Computer, Telecommunications and Information Technology, pp.365-368, 2011.

[14] W. Yanjun, C. Wen, and T. Chintha, "A PAPR Reduction Method Based on Artificial Bee Colony Algorithm for OFDM Signals", IEEE Transactions on Wireless Communications, Vol.9, No.10, pp.2994-2999, 2010.

[15] D. Karaboga and B. Basturk, "Artificial Bee Colony (ABC) Optimization Algorithm for Solving Constrained Optimization Problems", Foundations of Fuzzy Logic and Soft Computing, LNCS, No.4529, pp.789-798, 2007.

[16] N. Shi and W. Shouming, "A Partial Transmit Sequences Based Approach for the Reduction of Peak-to-Average Power Ratio in FBMC System", In: Proc. of Wireless and Optical Communications Conf., pp.1-3, 2016.

[17] D. Dardari, V. Tralli, and A. Vaccari, "A Theoretical Characterization of Nonlinear Distortion Effects in OFDM Systems", IEEE Transactions on Communications, Vol.48, No.10, pp.1764-1775, 2000. 